\title{
Normal childhood brain growth and a universal sex and anthropomorphic relationship to cerebrospinal fluid
}

\author{
Mallory R. Peterson, BS, ${ }^{1,2,6}$ Venkateswararao Cherukuri, PhD,, Joseph N. Paulson, PhD, ${ }^{7}$ \\ Paddy Ssentongo, MD, ${ }^{2}$ Abhaya V. Kulkarni, MD, PhD, ${ }^{8,9}$ Benjamin C. Warf, MD, ${ }^{10,11}$ \\ Vishal Monga, PhD, ${ }^{5}$ and Steven J. Schiff, MD, PhD ${ }^{1-4}$ \\ ${ }^{1}$ Center for Neural Engineering; Departments of ${ }^{2}$ Engineering Science and Mechanics, ${ }^{3}$ Neurosurgery, and ${ }^{4}$ Physics; ${ }^{5}$ School \\ of Electrical Engineering and Computer Science, The Pennsylvania State University, University Park; ${ }^{6}$ The Pennsylvania State \\ University College of Medicine, Hershey, Pennsylvania; ${ }^{7}$ Department of Biostatistics, Product Development, Genentech Inc., \\ South San Francisco, California; ${ }^{8}$ Department of Neurosurgery, University of Toronto; ${ }^{2}$ Department of Neurosurgery, The Hospital \\ for Sick Children, Toronto, Ontario, Canada; ${ }^{10}$ Department of Neurosurgery, Harvard Medical School; and ${ }^{11}$ Department of \\ Neurosurgery, Boston Children's Hospital, Boston, Massachusetts
}

\begin{abstract}
OBJECTIVE The study of brain size and growth has a long and contentious history, yet normal brain volume development has yet to be fully described. In particular, the normal brain growth and cerebrospinal fluid (CSF) accumulation relationship is critical to characterize because it is impacted in numerous conditions of early childhood in which brain growth and fluid accumulation are affected, such as infection, hemorrhage, hydrocephalus, and a broad range of congenital disorders. The authors of this study aim to describe normal brain volume growth, particularly in the setting of CSF accumulation.
\end{abstract}

METHODS The authors analyzed 1067 magnetic resonance imaging scans from 505 healthy pediatric subjects from birth to age 18 years to quantify component and regional brain volumes. The volume trajectories were compared between the sexes and hemispheres using smoothing spline ANOVA. Population growth curves were developed using generalized additive models for location, scale, and shape.

RESULTS Brain volume peaked at 10-12 years of age. Males exhibited larger age-adjusted total brain volumes than females, and body size normalization procedures did not eliminate this difference. The ratio of brain to CSF volume, however, revealed a universal age-dependent relationship independent of sex or body size.

CONCLUSIONS These findings enable the application of normative growth curves in managing a broad range of childhood diseases in which cognitive development, brain growth, and fluid accumulation are interrelated.

https://thejns.org/doi/abs/10.3171/2021.2.PEDS201006

KEYWORDS brain volume; cerebrospinal fluid; growth curves; hydrocephalus

$\mathrm{T}$ HE brain growth and cerebrospinal fluid (CSF) accumulation relationship is critical to characterize because it is impacted in numerous conditions of early childhood in which brain growth and fluid accumulation are affected, such as infection, hemorrhage, hydrocephalus, and a broad range of congenital disorders. ${ }^{1,2}$ Appropriate management of such diseases requires an understanding of the normal dynamics of brain growth in relationship to fluid accumulation. However, the study of brain size and growth has a long and contentious history. ${ }^{3,4}$ In the pre-magnetic resonance imaging (MRI) era, postmortem studies provided insight into brain volume changes over the human lifespan, but such methods suf- fered from inherent inaccuracies. ${ }^{5}$ In the MRI era, the development of advanced computational and statistical algorithms has enabled detailed in vivo volumetrics including components and regions of the brain. ${ }^{6-9}$

Nonetheless, studies defining normal brain volume growth patterns in the MRI era have often included small sample sizes, limited algorithm technology, incomplete coverage of the pediatric age range, retrospective cohorts of clinical patients, and a disparate array of curve fitting techniques..$^{6-8,10,11}$ Studies conducted by Pfefferbaum et al., Courchesne et al., and Giedd et al. included only 30, 50, and 243 subjects, respectively, in the pediatric age range. . $7,79^{-9}$ Moreover, none of those studies included the entire pedi-

ABBREVIATIONS ACR = American College of Radiology; CAT12 = Computational Anatomy Toolbox 12; CSF = cerebrospinal fluid; dHCP = Developing Human Connectome Project; GAMLSS = generalized additive models for location, scale, and shape; MDI = Mental Development Index; $\mathrm{MRI}=$ magnetic resonance imaging; NIH = National Institutes of Health; SPM12 = Statistical Parametric Mapping 12; SSANOVA = smoothing spline ANOVA; WASI = Wechsler Abbreviated Scale of Intelligence; WHO = World Health Organization.

SUBMITTED December 29, 2020. ACCEPTED February 19, 2021.

INCLUDE WHEN CITING Published online July 9, 2021; DOI: 10.3171/2021.2.PEDS201006. 
atric age range from birth to age 18 years. More recent works such as those by Hedman et al. and Coupé et al. have incorporated big data or meta-analysis approaches, but by incorporating such large amounts of data, they lose rigor in exclusion criteria, population representation, and calibration between scan site acquisition. ${ }^{12,13}$ Furthermore, despite leveraging 56 studies and including 2211 subjects, Hedman et al. did not involve any subjects under the age of 4 years, excluding the period during which the majority of brain growth takes place. ${ }^{12}$ Others such as Knickmeyer et al. have looked closely at the infantile period from birth to age 2 years but have not covered the later pediatric ages. ${ }^{14}$

Despite the enormous amount of recent interest, a definitive set of normative childhood brain growth percentile curves, such as those we have for multiple other anthropomorphic measures like weight, height, and head circumference, are not yet available. Furthermore, the interaction of brain growth and the regulation of the fluid compartment within which it is buoyant and immersed during development has not been well characterized. Herein, we resolve both of these open issues.

\section{Methods \\ Cohort Characteristics}

The MRI scans used in this study were taken from the National Institutes of Health (NIH) Pediatric MRI Repository (https://nda.nih.gov) under an institutional data use agreement between The Pennsylvania State University and the National Institute of Mental Health, approved on January 14, 2019. This repository was developed using a scaled-down US census from 2000 (to appropriately represent the racial and socioeconomic demographic characteristics of the entire US pediatric population) and included rigorous exclusion criteria (described in the Supplemental Material) to ensure healthy participants with normal brain development. The repository aimed to obtain 2-year longitudinal follow-up scans for individual participants and was able to accomplish this for 378 of the 505 total subjects, making this a cross-sequential study. ${ }^{15}$ The cross-sequential format is ideal for the development of growth curves. ${ }^{7,16}$ The number of subjects in this study was 505 (259 females), with a total of 1067 MRI scans given the longitudinal nature of the cohort. The minimum age was 13 days, and the maximum age was 22 years, but only scans from subjects up to 18 years old were included to develop growth curves representative of the pediatric age range. Scans existed for participants in each year of life throughout the pediatric age range, as seen in Fig. 1.

\section{MRI Acquisition and Quality Control}

The NIH Pediatric MRI Repository database was collected at Boston Children's Hospital, Cincinnati Children's Hospital Medical Center, The University of Texas Medical School at Houston, Resnick Neuropsychiatric Hospital at UCLA, Children's Hospital of Philadelphia, and Washington University in St. Louis. While it is beneficial to use multiple different geographic locations when cultivating a cohort representative of a particular population that can then be leveraged for growth curves, bias can be introduced through the use of different scanner sites.
MRI conditions such as scanner manufacturer, acquisition protocols, and field strength can all contribute to the underlying compatibility of MRI data gathered at different sites. For the NIH Pediatric MRI Repository, the acquisition protocols were well defined and reproduced uniformly at $1.5 \mathrm{~T}$ at each site. ${ }^{15}$ Additional acquisition details can be found in the Supplemental Material.

Because different MRI scanners were used, the same healthy person was scanned at each site to serve as a calibrating "living phantom." ${ }^{17}$ In order to ameliorate the intersite differences and characterize the gradient distortion field, the investigators applied a geometrical distortion correction using a 3D American College of Radiology (ACR) phantom. The ACR phantom scanning was completed approximately monthly, and the living subject scanning was completed annually to ensure intersite calibration.

Regarding quality control of the data set, each MRI scan within the repository was viewed by a radiologist to ensure no neurological abnormalities, and the scans were then assessed for quality. If the first scan had too much motion artifact, it was redone. If the scan was deemed to be neurologically normal and of acceptable quality, only then was it included in the database.

In our present work with this database, all 1381 available anatomical MRI scans from the database were analyzed. In 314 of the 1381 scans, our final segmentation algorithms failed to successfully generate segmentation without computational error, and these scans were removed from further consideration. Each remaining volume segmentation was then visually audited to ensure reliable results. No manual editing was required.

\section{Segmentation Algorithms}

Currently, no single algorithm exists that can reliably segment the brains of both young infants and older subjects. ${ }^{18,19}$ This is particularly attributable to the myelination changes that do not resolve until approximately 2 years of age. ${ }^{20}$ These myelination changes lead to difficulty in establishing intensity thresholds among gray matter, white matter, and CSF. In order to maximize thresholding intensities, neonatal segmentation techniques most commonly rely on T2-weighted MRI scans rather than the T1-weighted scans, which are the predominant scan type used in older cohort segmentation algorithms. For these reasons, two different algorithms were used in this study.

The neonates and infants were assessed using the Developing Human Connectome Project (dHCP) pipeline, which required T2-weighted images. dHCP pipeline is freely available (https://github.com/BioMedIA/dhcpstructural-pipeline).

The older subjects were assessed with the Computational Anatomy Toolbox 12 (CAT12) within the Statistical Parametric Mapping 12 (SPM12) platform using Matlab 2019b, which relies on T1-weighted images. ${ }^{21}$ This software utilizes voxel and region-based morphometry upon application of an adaptive probability regiongrowing (APRG) algorithm for skull stripping. ${ }^{21}$ Each of the resulting scan sets was visually inspected to ensure that appropriate skull stripping and segmentation was accomplished, with no manual segmentation required. Once the volumes determined by each segmentation procedure 


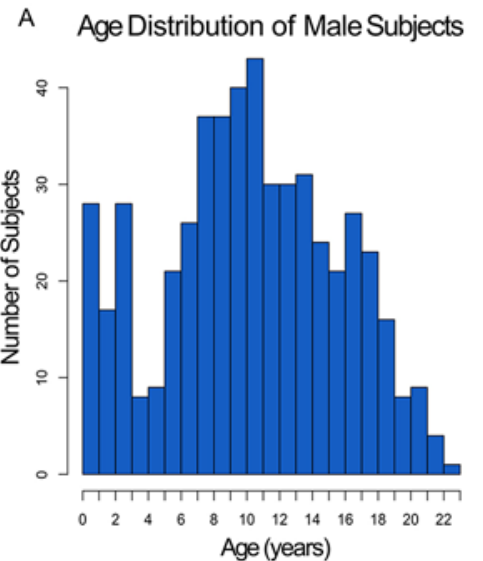

B Age Distribution of Female Subjects

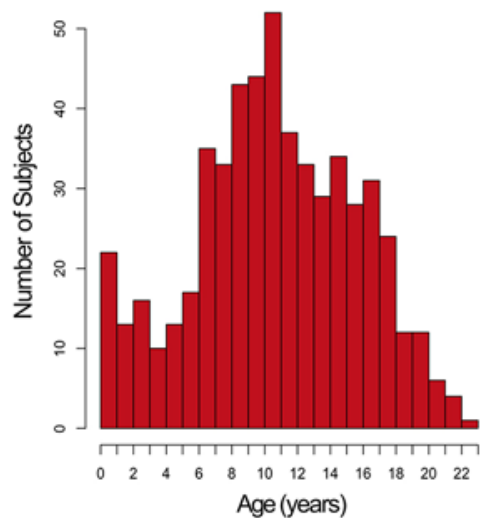

\begin{tabular}{|l|l|l|l|}
\hline & Female & Male & Total \\
\hline Scans & 549 & 518 & 1067 \\
\hline Subjects & 259 & 246 & 505 \\
\hline Min Age (days) & 14 & 13 & NA \\
\hline Max Age (days) & 8131 & 8073 & NA \\
\hline
\end{tabular}
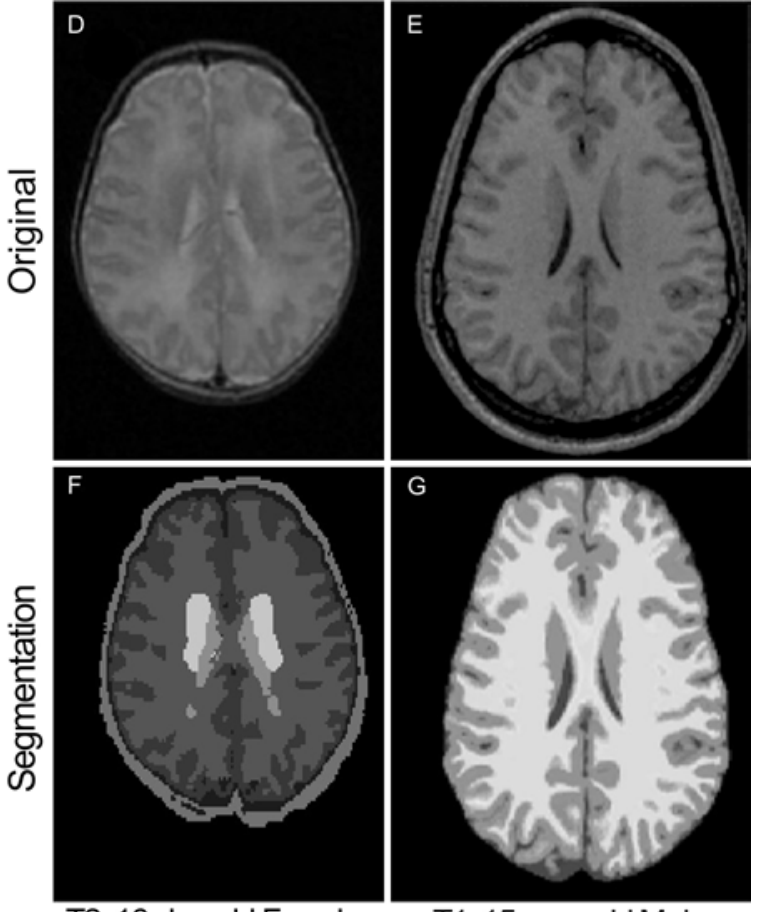

T1: 15-year-old Male

FIG. 1. Data from each age group from 13 days old to 22 years old were included in the cohort for both males (A) and females (B). The cross-sequential study included 505 total subjects (C), with most having between 2 and 3 longitudinal visits (separated by 2 years). For this study, only scans from 13-day-old to 18-year-old subjects were included to focus on the pediatric age range. Two different algorithms for volume quantification were used, one for the neonates and one for the older cohort. As an example, a T2-weighted MRI scan (D) from a 16-day-old female is shown. This scan was processed through the dHCP pipeline to produce a segmentation (F) including gray matter (found by adding the deep gray matter and cortical gray matter), CSF, and white matter. A T1-weighted MRI scan (E) from an older patient (15-year-old male) is shown. This scan was processed using the CAT12 pipeline within SPM12 to produce the segmentation $(\mathbf{G})$ including gray matter, CSF, and white matter. Figure is available in color online only.

were established, the accompanying atlases were used to compile volumes for the desired regions from smaller sections of the brain.

\section{Statistical Analysis: Smoothing Spline ANOVA}

One of the major limitations of the historical fitting efforts is the inability to statistically assess which time points throughout the entire growth process are truly different between groups while also establishing appropriate representative trajectories. Within this study, we split our efforts, using one approach for trajectory modeling and a different approach for statistical analysis. The generalized additive models for location, scale, and shape (GAMLSS) approach was used to provide smooth trajectory growth curves and centiles for reference in the same manner in which the World Health Organization (WHO) produces their growth curves. However, these curves do not facilitate an in-depth statistical analysis of differences between groups such as the two different sexes or hemispheres. Therefore, the smoothing spline ANOVA (SSANOVA) technique was also incorporated into this study for the main purpose of examining longitudinal statistical differences between populations. The smoothing parameters are determined through an iterative cross-validation process, which is then followed by the minimization of the penalized least square functional, whose solution is a smoothing spline. The details of this process are described in the book by $\mathrm{Gu}^{22}$ and are more fully described in the Supplemental Material.

For this study, the component differences between males and females were explored within the $\mathrm{R}$ platform (R Foundation for Statistical Computing) using nonparametric SSANOVA mixed-effects models with a subjectspecific random-effects component added to account for the cross-sequential aspect of the data. ${ }^{22}$ The model included age and sex or hemisphere as main factors, as well as an interaction term. We were interested in visualizing and statistically analyzing the longitudinal differences between hemispheres and therefore incorporated hemisphere as a main factor rather than a nested factor. Time periods with significant sex or hemispheric differences were defined as regions at which there was no overlap between the Bayesian 95\% confidence intervals calculated for the sex and hemispheric factors. These regions of significant difference were highlighted on the plotted models, and the time period of significance was documented as well.

\section{Statistical Analysis: GAMLSS}

The smooth growth curves used to fit the volumes and 
other growth metrics included in this study were developed using the GAMLSS software implemented in R. ${ }^{23}$ The Box-Cox power exponential distribution, which was chosen by the WHO for their standard growth curves, was used to model the volumes in this study. ${ }^{24}$ For the total brain tissue growth curves, we utilized data for subjects between 18 and 22 years of age to set the 18 -year-old volume intercepts and the perinatal volume data from Hüppi et al. to set the volume intercepts at birth. ${ }^{25}$

To determine the peaks of the brain tissue and gray matter curves, the cftool within Matlab 2019b was used to fit differentiable rational polynomial functions (which were applied as the smoothing function in the GAMLSS curves). The significant sex and hemispheric differences were found using the Mann-Whitney U-test within Matlab 2019b. A Bonferroni correction was applied so that significance was established with $\mathrm{p}<0.000066$.

The weight-for-height and height-for-age normalizations were accomplished by fitting a GAMLSS curve to the weight-for-height and height-for-age data from the NIH repository for each sex. Based on these fits, percentiles were calculated for each subject, and the 50th percentile was set at 1 , with percentiles above and below ranging from 0.5 to 1.5 . The corresponding brain volume for each subject was then divided by this percentile value to obtain normalized brain volumes.

\section{Statistical Analysis: Cognitive Score Correlations}

Cognitive scoring was included in the NIH Pediatric MRI Repository study, with Wechsler Abbreviated Scale of Intelligence (WASI) tests undertaken in participants ranging from 6 to 18 years of age. The infants (from birth to 3 years of age) were assessed using the Bayley Scales of Infant Development, Second Edition (BSID-II) Mental Development Index (MDI). We fit a linear mixed-effects model (with subject identification as the random-effects component) to the appropriate cognitive score using the brain volume $\mathrm{z}$-score values. While the linear fits showed positive slopes for each metric, only the WASI scores showed a significant fixed effect for the brain volume zscore. Age-dependent correlations were calculated with a subpopulation window size of 120 and step size of 20 subjects for raw brain volume, brain volume z-score, and weight-for-height normalized volume z-score.

\section{Data Availability}

The volumes and all data used to create the SSANOVA and GAMLSS growth curves presented here are provided in a spreadsheet supplied in the Supplemental Extended Data.

\section{Code Availability}

The code and data needed to reproduce the figures in this study, as well as plot new volumes on the existing growth curves or calculate z-scores, are uploaded to the Github site (https://github.com/Schiff-Lab/Brain-Growth).

\section{Results}

We quantified brain and CSF volumes from 1067 brain
MRI scans from 505 healthy subjects (259 females) from birth to age 18 years. These MRI scans were collected from healthy pediatric participants in the NIH Pediatric MRI Repository, which was accessed under an institutional data use agreement between The Pennsylvania State University and the National Institute of Mental Health. This MRI repository was developed based on a scaleddown US census with rigorous exclusion criteria, with the goal of providing a standard representation of the socioeconomic, male and female, and racial distribution of healthy normal US children..$^{15}$ The cross-sequential cohort contains participants in each year of life ranging from 13 days to 22 years old (Fig. 1A-C). Uniform MRI scanning protocols were applied at each data collection site, and living phantom calibration was leveraged to ensure volumetric consistency between sites. ${ }^{15,17}$

The T2-weighted MRI scans of the neonatal and infant population were processed using dHCP pipeline to appropriately segment the rapidly growing and incompletely myelinated brains. ${ }^{26}$ The T1 images of the older subjects were processed using the CAT12 within the SPM12 software. Each of the resulting segmentation images (Fig. $1 \mathrm{D}-\mathrm{G})$ were manually inspected to confirm appropriate anatomical assignment.

The brain components and region volumes quantified by the $\mathrm{AHCP}$ and CAT12 pipelines were then fit using SSANOVA with random effects (to account for the crosssequential design of the study) to define time periods of statistically significant differences stratified by sex and hemisphere (Figs. 2-4). ${ }^{22}$ In order to define the growth trajectories and population centiles, the volumes were also fit using GAMLSS with a Box-Cox power exponential distribution (Supplemental Figs. 1 and 2), which is the same platform and distribution leveraged by the WHO to develop their standard growth curves for weight, height, and head circumference. ${ }^{23,27}$ Finally, the relationship between cognitive scores and brain volume was explored using a linear mixed-effects model and age-dependent correlations (Fig. 5 and Supplemental Fig. 3).

\section{Findings Differentiated by Sex}

Males exhibited larger overall brain volumes than females throughout childhood (Fig. 2A). The volume for females peaked at 10.7 years, and the volume for males peaked at 11.2 years, followed by a slow but consistent decrease. This early adolescent peak has been previously described ${ }^{10,12} \mathrm{CSF}$ increased throughout childhood, with male fluid accumulation significantly larger than that for females after the 3rd year of life (Fig. 2B). Gray matter (Fig. 2C) peaked at 7.5 years for males and 7.4 years for females and then began to gradually decrease, whereas white matter (Fig. 2D) continued to progressively increase into early adulthood. ${ }^{28}$ The ratio of gray/white matter (Fig. 2E) showed an initial increase, peaking before 2 years of age, followed by a progressive decrease thereafter. Female gray/white matter ratios were significantly larger than male ratios between ages 9 and 11, although the difference was small (Fig. 2E).

We performed body size normalization to assess if differences in brain volume persisted between males and females. Allometry, or differential growth, of the brain with 


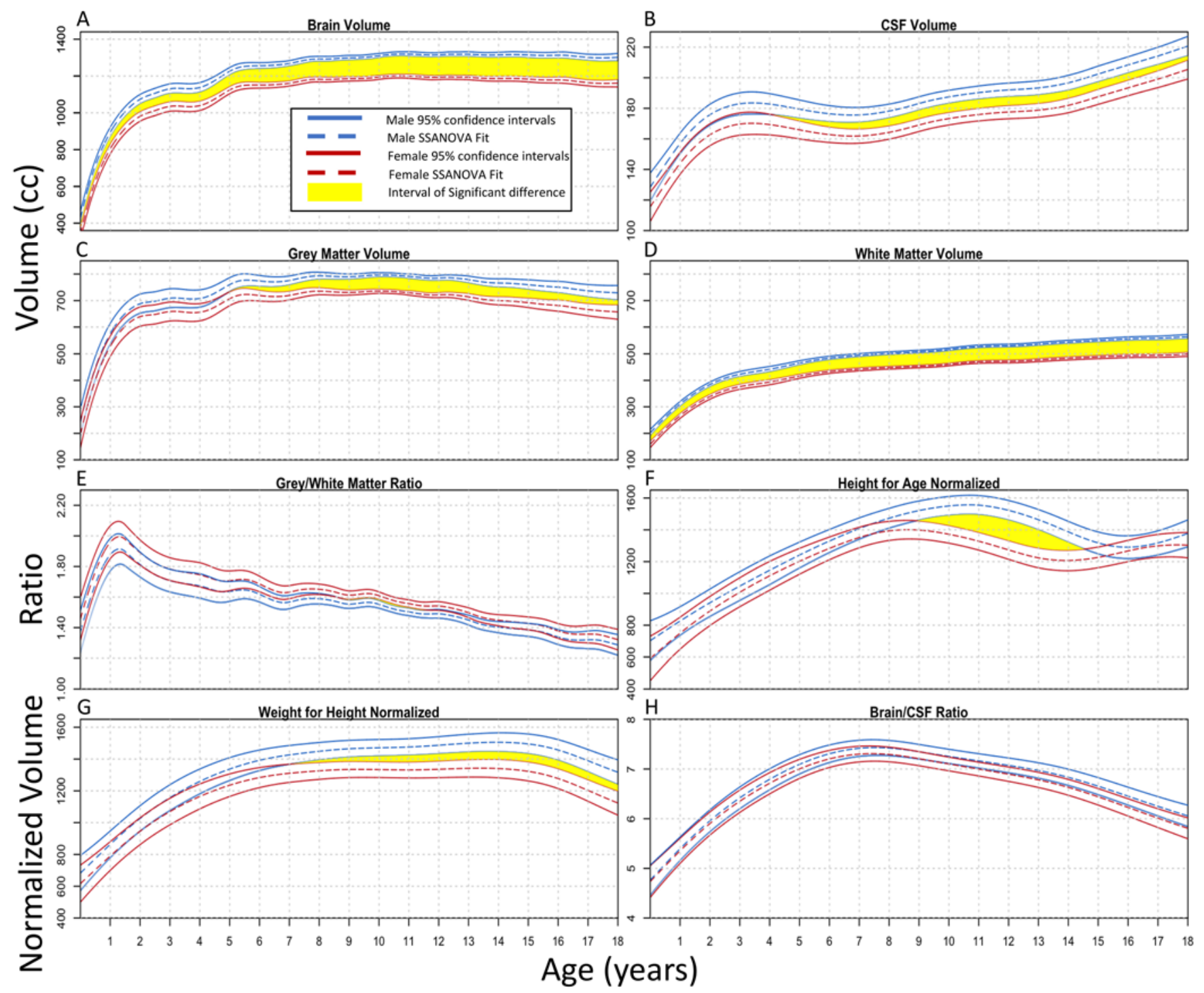

FIG. 2. SSANOVA models with random effects were fit to total brain volume (A), CSF volume (B), gray matter volume (C), white matter volume $(\mathbf{D})$, gray/white matter ratio $(\mathbf{E})$, height-for-age normalized brain volume $(\mathbf{F})$, weight-for-height normalized brain volume $(\mathbf{G})$, and brain/CSF ratio $(\mathbf{H})$. For each plot, the male data are shown in blue and the female data in red. The dashed line represents the fit, with the two solid lines on either side showing the Bayesian 95\% confidence intervals. The upper and lower intervals for the right and left sides overlap throughout the entire time frame for the brain/CSF ratio; therefore, there are no statistically significant differences at any age. For the other plots, time periods of significant differences where the intervals do not overlap are shown in yellow.

respect to body size was discussed in depth by D'Arcy Wentworth Thompson in 1917 in On Growth and Form.29 Gould, in The Mismeasure of Man, attempted and failed to eliminate sex differences through body size normalization. ${ }^{4}$ Nevertheless, much of the volumetric brain study in the MRI era has not accounted for anthropomorphic normalization. Figure $2 \mathrm{~F}$ and $\mathrm{G}$ shows brain volume normalized by height-for-age and weight-for-height, respectively, which did not eliminate the sex-based differences in volume. Muscle mass content, greater in males, has been correlated with larger brain volumes, but not with higher cognitive capability. ${ }^{30}$ However, the ratio of brain/ CSF volume demonstrated no significant sex differences at any age, without requiring anthropomorphic normalization (Fig. 2H).

\section{Hemispheric Lateralization Findings}

Figure $3 \mathrm{~A}$ and $\mathrm{B}$ shows that there was no lateralizing difference in size between right and left hemispheres for males and females. Figure 3C and D shows that the left temporal lobe was significantly larger than the right for both males and females, which has been controversial in evaluating intractable epilepsy. ${ }^{31}$ In addition, the hippocampi were significantly larger on the right than the left side for both sexes (Fig. 3E and F). Previous studies, such as the one by Lynch et al., have also found that the right 

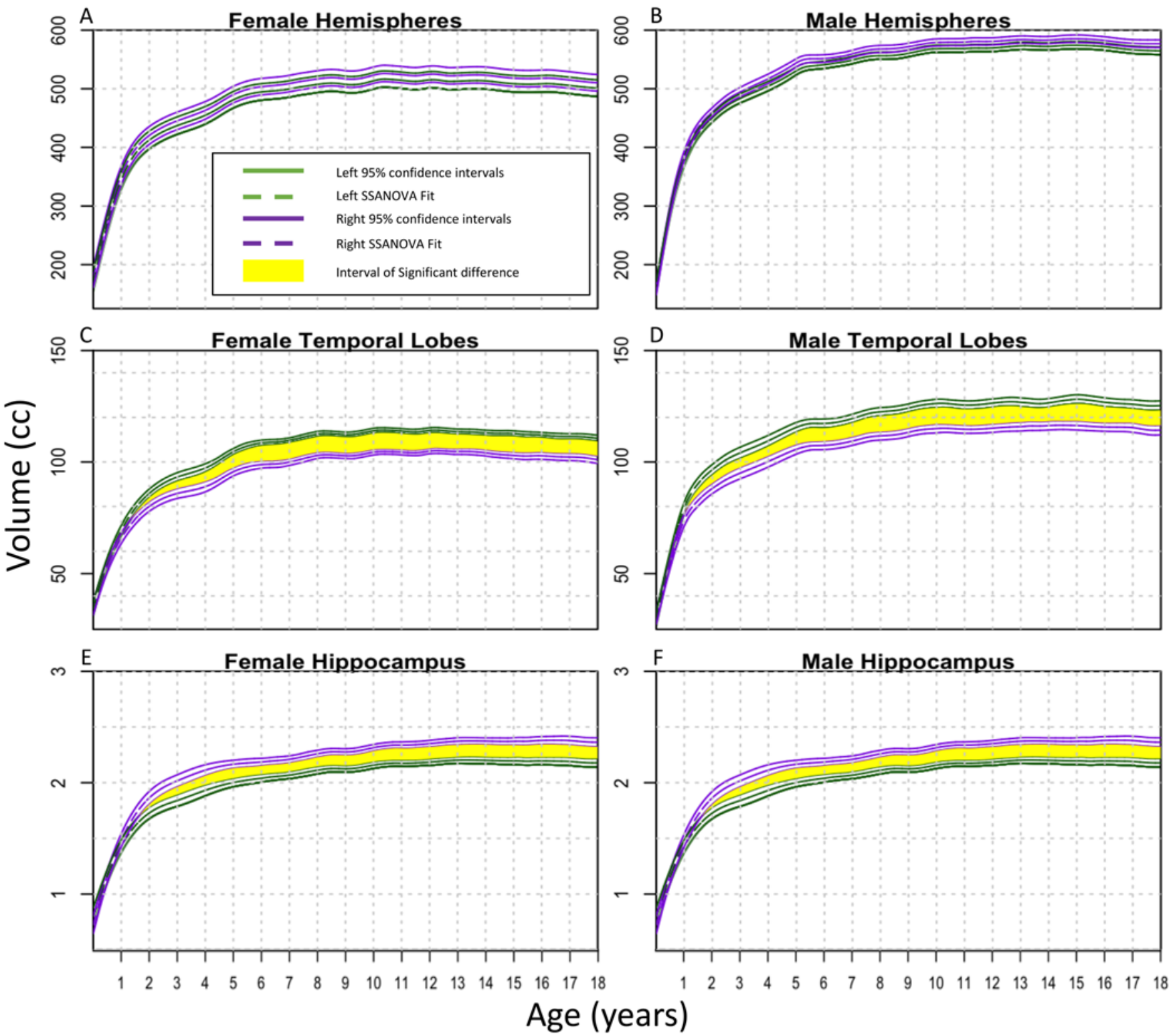

\section{Age (years)}

FIG. 3. SSANOVA models with random effects were fit to hemispheres (A and $B)$, temporal lobes ( $C$ and $D$ ), and hippocampi $(\mathrm{E}$ and $\mathrm{F})$. For each plot, the left side is represented by green and the right side by purple. The dashed line represents the fit, with the two solid lines on either side showing the Bayesian 95\% confidence intervals. The upper and lower intervals for the right and left sides overlap throughout the entire time frame for the hemispheres; therefore, there are no statistically significant differences. For the temporal lobes and hemispheres, time periods of significant differences at which the intervals do not overlap are shown in yellow.

hippocampus is larger than the left. ${ }^{32}$ There were no lateralizing differences for the volumes of male and female frontal lobes, parietal lobes, occipital lobes, or cerebella (Fig. 4A-H).

\section{Correlation Between Cognition and Volume}

Cognitive scores showed a small but significant correlation with brain volume in the 4 years leading up to the peak in volume. The MDI scores for infants from birth to age 3 years were not significantly predicted by brain volume z-scores (Fig. 5B), but the WASI scores for ages 6-18 years were significantly correlated with brain volume $\mathrm{z}$-scores (Fig. 5A), as described previously. ${ }^{33}$ The correlation between cognitive score and brain volume was significant for raw brain volume, age-adjusted brain volume z-score, and weight-for-height normalized volumes in the years immediately preceding the brain volume peak (Fig. 5C-E). This correlation was not maintained when separating into smaller cohorts by sex (Supplemental Fig. 3).

\section{Growth Curves and Centiles}

In 1987, Roche et al. created head circumference growth curves from studying 888 healthy US children, ${ }^{34}$ 


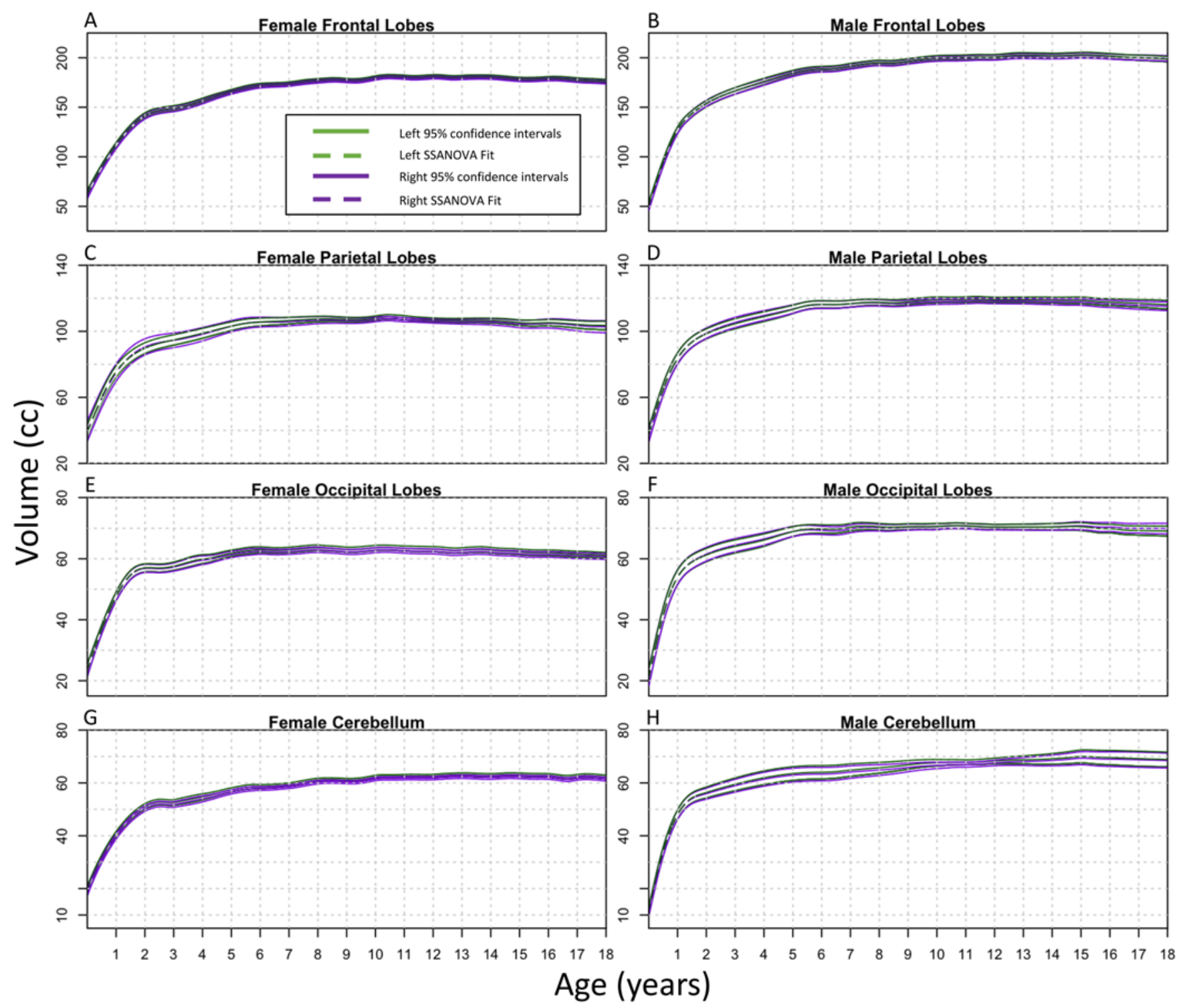

FIG. 4. SSANOVA models with random effects were fit to frontal lobes ( $A$ and $B)$, parietal lobes ( $C$ and $D)$, occipital lobes ( $E$ and $F)$, and cerebella ( $\mathbf{G}$ and $\mathbf{H})$. For each plot, the left side is represented by green and the right side by purple. The dashed line represents the fit, with the two solid lines on either side showing the Bayesian 95\% confidence intervals. The upper and lower intervals for the right and left sides overlap throughout the entire time frame for each region; therefore, there are no statistically significant differences.

and such normative head circumference curves from US ${ }^{35}$ and $\mathrm{WHO}^{27,36}$ cohorts are now used in routine clinical practice as indirect metrics of brain growth. Figure 6 illustrates the analogous GAMLSS pediatric brain volume growth curves for males and females (Fig. 6A and B) together with early brain volume growth and CSF volume insets, as well as the brain/CSF ratio (Fig. 6C and D) for a more comprehensive presentation of childhood normative brain growth suitable for clinical use.

\section{Discussion}

Brain volume measurement became a field of study of biological determinism pioneered by Samuel Morton in the mid $1800 s^{4,37}$ Morton filled over 1000 cranial vaults with mustard seed and lead shot to determine brain volume, which he then compared between races and genders. A century and a half later, Gould used Morton as a case study in scientific bias. ${ }^{4}$ Decades after the publication of Gould's analyses in The Mismeasure of Man, arguments over biases and flaws continue in the assessment of the volume of the normal human brain. ${ }^{3}$

The sexual dysmorphism found in this present study corroborates previous studies looking at different age groups. Giedd et al., Coupé et al., Nopoulos et al., and Lenroot et al. all found similar differences in brain components, with larger total brain volumes in males., ${ }^{9,13,38,39}$ 

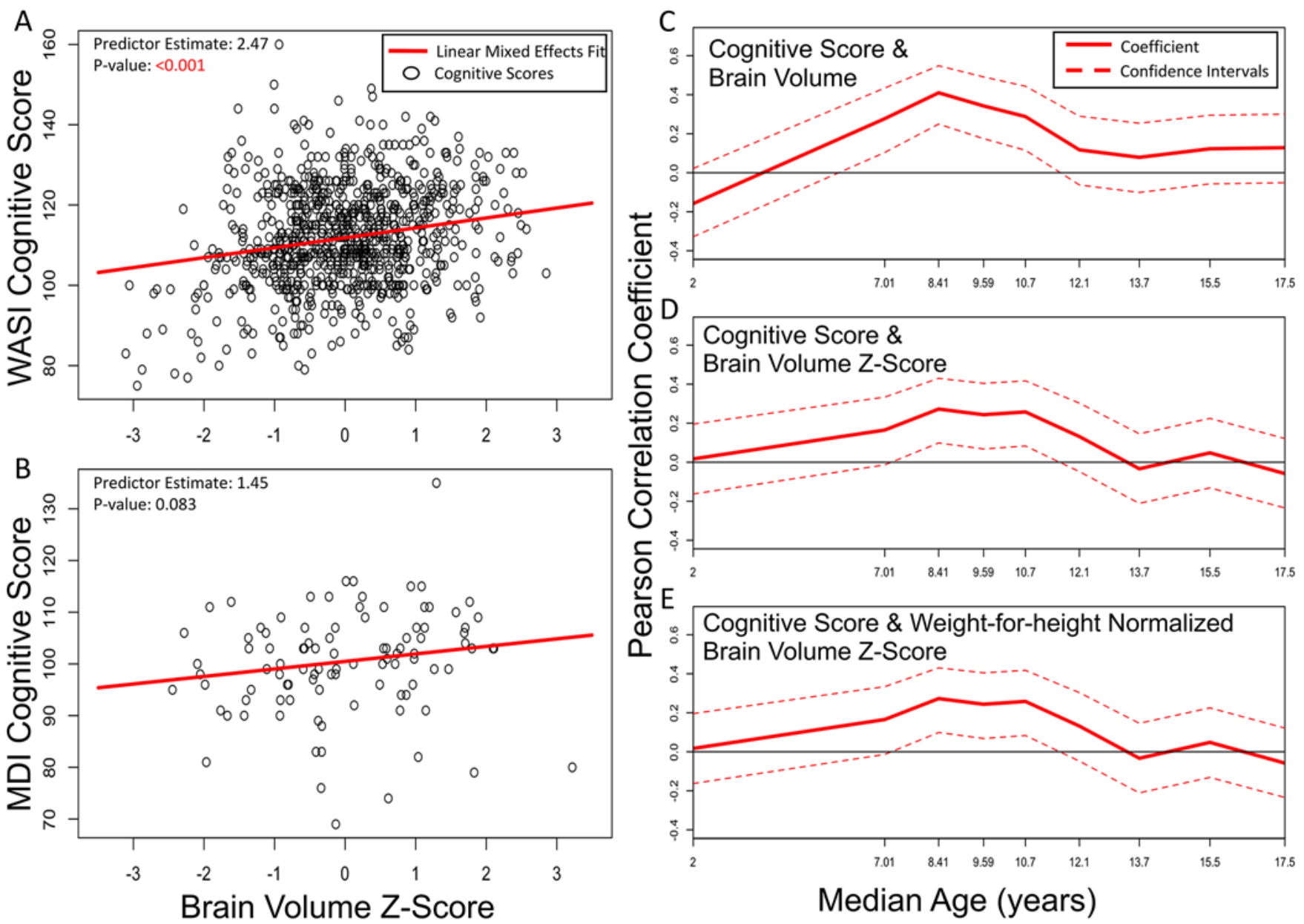

FIG. 5. Two cognitive scores were used, the MDI for infants from birth to age 3 years and the WASI tests for adolescents aged 6 to 18 years. Linear mixed-effects models were fit for the relationship between WASI and brain volume z-score $(\mathbf{A})$ as well as the relationship between MDI and brain volume z-score (B). Age-dependent correlations were calculated with a subpopulation window size of 120 and step size of 20 subjects to show the Pearson correlation between cognitive score and raw brain volume (C), brain volume z-score (D), and weight-for-height normalized brain volume z-score $(E)$ at different ages. Figure is available in color online only.

We found that normalizing by body measurements did not account for sex differences. Interestingly, Coupé et al. used proportionalization by total intracranial volume and failed to find an overall sex difference, ${ }^{13}$ a finding consistent with our discovery of a universal age-dependent ratio between brain and CSF volumes.

Another area of great interest in brain volume research is the growth trajectory of components of the brain. Head size increases steadily until at least age 18 years. ${ }^{34}$ In the MRI era, however, many studies have shown that total brain volume reaches its peak in earlier adolescence. Without including younger children during the early years of rapid brain growth, the location of this volume peak can be somewhat uncertain. Giedd et al. and Hedman et al. showed that brain volume peaks between ages 12 and 14 years, but they lacked subjects below age 4 years, when the majority of brain growth takes place.,12 Lenroot et al. found peaks at age 10.5 years for females and 14.5 years for males but lacked subjects below the age of 3 years. ${ }^{39}$ Incorporating more children during the rapid early growth of the first few years of life, our analysis placed the peak at $10-12$ years of age.

It is interesting to note that Hedman et al. found an early adolescent peak but also concluded that their metaanalysis highlighted a possible later resurgence of growth with a secondary growth peak in adulthood. ${ }^{12}$ These findings highlight the need for additional normal growth data throughout the human lifespan. Such lifespan data would gain greater value by incorporating fetal through postnatal volume development, much in the manner of Holland et al. ${ }^{40}$

Whereas total brain volume peaked in adolescence, we found gray matter to peak earlier and then slowly decrease. Our results showed that gray matter peaked in the 7 th year of life for both males and females. Sowell et al. also found that gray matter decreased after the 7th year of life, but they did not include any subjects under the age of $7{ }^{41}$ While gray matter peaks in late childhood and starts to decline thereafter, the white matter and CSF components do not reach a peak during the pediatric age range. 


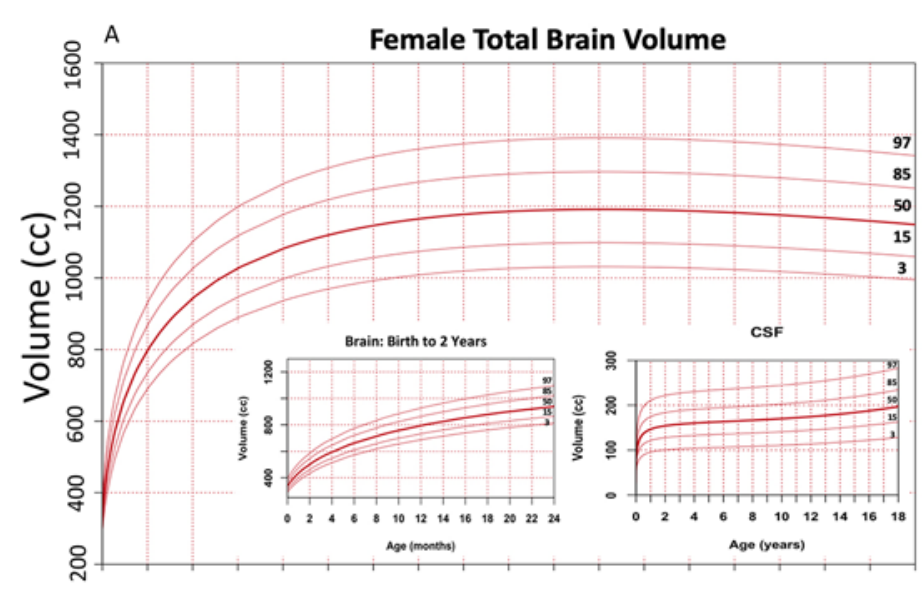

B Male Total Brain Volume

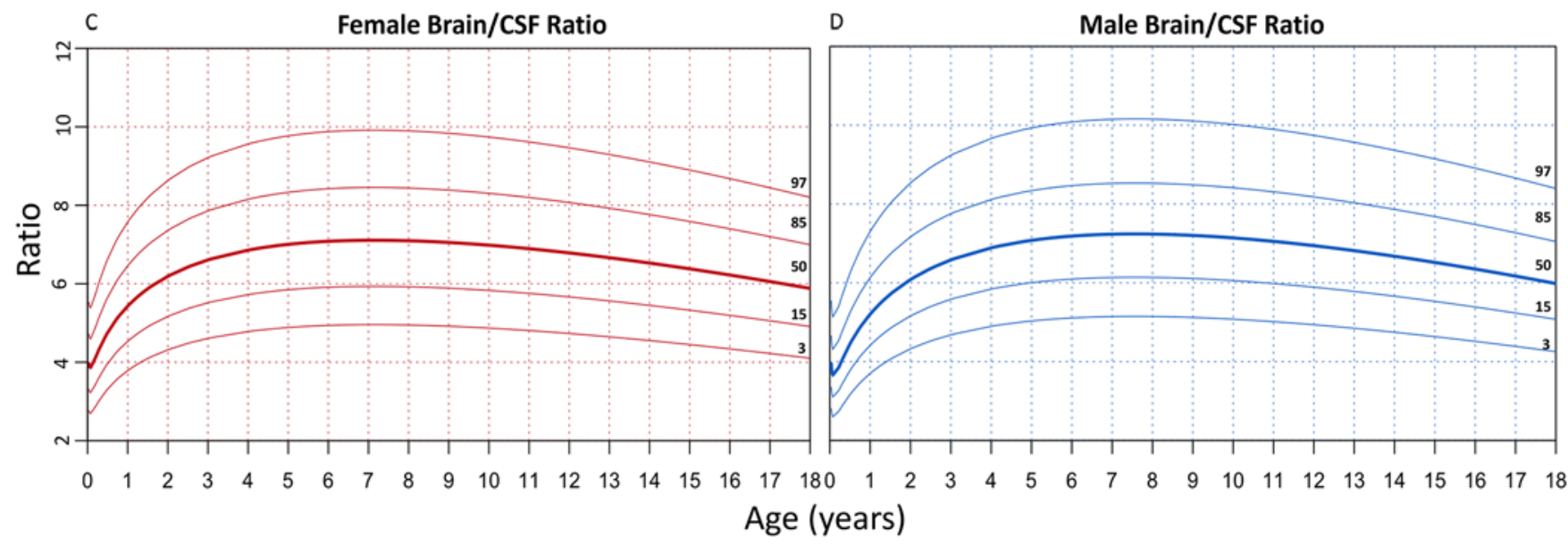

FIG. 6. To provide a standard tool for researchers and clinicians, normal curves resembling head circumference growth curves were fit using GAMLSS to show the 3rd, 15th, 50th, 85th, and 97th percentiles of normal brain volume growth for females (A) and males (B), as well as the brain/CSF ratio for females (C) and males (D). The growth curves were modeled using a Box-Cox power exponential distribution and smoothed using fractional polynomials. Both male and female brain volume plots include insets for brain growth from birth to age 2 years, as well as CSF accumulation from birth to age 18 years, in order to provide a comprehensive picture of brain growth dynamics in the pediatric age range. Figure is available in color online only.

Sowell and others have found that white matter reaches a peak near the age of 40, whereas CSF accumulation never ceases. $^{28,41}$ It is appreciated that myelination continues through the 2 nd decade of life, contributing to white matter growth. ${ }^{42,43}$ Gray matter, however, undergoes synaptic elimination through pruning, which may account for the peak seen in late childhood. ${ }^{4}$

It is after the gray matter peak at 7 years of age that we found a significant correlation between brain volume and cognitive scoring. This correlation continued until the total brain volume peaked near age 12 . These findings highlight this critical period of pruning and myelination before overall brain volume begins to decline in late childhood. In previous work, our group examined candidate growth models for the brain. ${ }^{8}$ One of the study limitations of that work was the small sample size. In this present study, we have exhaustively incorporated what is, to our knowledge, the largest, rigorously selected data set of healthy children's normal MRI scans. Constructing percentile growth curves for clinical use, as opposed to model fitting for biology, requires accurate nonlinear curve fitting without re- gard to representing the underlying biology. ${ }^{34}$ Figure 5 is, to our knowledge, the most definitive set of normal brain growth percentile curves suitable for clinical use, along with percentile curves for the brain/CSF ratio. Because it is currently unknown how to best incorporate these growth curves in clinical practice, we recommend using them within research protocols until best practices can be determined. We archive a high-quality growth chart in the Supplemental Material to facilitate reproduction.

While the subjects for this study were drawn from low, middle, and high socioeconomic status categories using US Department of Housing and Urban Development methods for family income adjusted for region and family size, this study did not explore the impact of social determinants of health on brain growth. ${ }^{15}$ Previous works, such as that by Decker et al., have found correlations among family income, cognitive function, and anterior hippocampus volume..$^{45}$ It is very possible that social determinants of health play a role in differential brain growth in other regions of the brain, and future studies should continue to explore this relationship. 
Our study has limitations. While the growth curves developed in this study provide a standard representation of the US pediatric population, they can only act as a reference and not as a standard for other geographic regions. Other growth curves specific to particular regions and peoples should be developed using healthy cohorts derived from those regions in order to provide appropriately representative standards, representing the diversity and characteristics of non-US populations.

Our findings demonstrate that for a broad spectrum of human disease affecting neurocognitive development and brain growth-ranging from hydrocephalus to neonatal sepsis, intraventricular hemorrhage of prematurity, childhood malnutrition, and epilepsy-measuring brain growth and CSF with respect to normative values is now feasible. The apparent universal nature of the age-dependent brain/ CSF ratio, regardless of sex or body size, suggests that the role of this ratio offers novel ways to characterize conditions affecting the childhood brain.

\section{Conclusions}

We have produced, to our knowledge, the first set of normal brain volume growth percentile curves from a healthy cohort representative of the US pediatric population. These growth curves show that while male and female brain growth is different, the ratio of brain tissue/ CSF is conserved across anthropomorphic measurements and sex.

\section{Addendum}

It has come to the attention of the authors that since this article has been in press, substantial work is being done on expanding the use of brain volume growth curves to include the entire lifespan. The following article has been posted as a preprint but is not yet peer reviewed: Bethlehem RAI, Seidlitz J, White SR, et al. Brain charts for the human lifespan. bioRxiv. 2021; https://doi. org/10.1101/2021.06.08.447489.

\section{Acknowledgments}

We are grateful to T. Sauer and S. Sinnar for helpful discussion and to Y. Wang and J. Chai for technical help in compiling data. This research was supported by the Penn State and National Science Foundation Center for Healthcare Organization Transformation collaboration, the NIH (grant no. R01HD085853: V.C., A.V.K., B.C.W., V.M., S.J.S.; and grant no. 1F30HD102120: M.R.P.), and the NIH Director's Transformative Award (grant no. R01AI145057: J.N.P., S.J.S.).

\section{References}

1. Kulkarni AV, Schiff SJ, Mbabazi-Kabachelor E, et al. Endoscopic treatment versus shunting for infant hydrocephalus in Uganda. N Engl J Med. 2017;377(25):2456-2464.

2. Jin SC, Dong W, Kundishora AJ, et al. Exome sequencing implicates genetic disruption of prenatal neuro-gliogenesis in sporadic congenital hydrocephalus. Nat Med. 2020;26(11):1754-1765.

3. Mismeasure for mismeasure. Nature. 2011;474(7352):419419.

4. Gould SJ. The Mismeasure of Man. Norton; 1981.

5. Dekaban AS. Changes in brain weights during the span of human life: relation of brain weights to body heights and body weights. Ann Neurol. 1978;4(4):345-356.
6. Pfefferbaum A, Mathalon DH, Sullivan EV, et al. A quantitative magnetic resonance imaging study of changes in brain morphology from infancy to late adulthood. Arch Neurol. 1994;51(9):874-887.

7. Courchesne E, Chisum HJ, Townsend J, et al. Normal brain development and aging: quantitative analysis at in vivo MR imaging in healthy volunteers. Radiology. 2000;216(3):672682.

8. Peterson M, Warf BC, Schiff SJ. Normative human brain volume growth. J Neurosurg Pediatr. 2018;21(5):478-485.

9. Giedd JN, Blumenthal J, Jeffries NO, et al. Brain development during childhood and adolescence: a longitudinal MRI study. Nat Neurosci. 1999;2(10):861-863.

10. Giedd JN, Snell JW, Lange N, et al. Quantitative magnetic resonance imaging of human brain development: ages 4-18. Cereb Cortex. 1996;6(4):551-560.

11. McAllister A, Leach J, West H, et al. Quantitative synthetic MRI in children: normative intracranial tissue segmentation values during development. AJNR Am J Neuroradiol. 2017;38(12):2364-2372.

12. Hedman AM, van Haren NE, Schnack HG, et al. Human brain changes across the life span: a review of 56 longitudinal magnetic resonance imaging studies. Hum Brain Mapp. 2012;33(8):1987-2002.

13. Coupé P, Catheline G, Lanuza E, Manjón JV. Towards a unified analysis of brain maturation and aging across the entire lifespan: a MRI analysis. Hum Brain Mapp. 2017;38(11):5501-5518.

14. Knickmeyer RC, Gouttard S, Kang C, et al. A structural MRI study of human brain development from birth to 2 years. $J$ Neurosci. 2008;28(47):12176-12182.

15. Evans AC. The NIH MRI study of normal brain development. Neuroimage. 2006;30(1):184-202.

16. Diggle PJ, Heagerty P, Liang KY, Zeger SL. Analysis of Longitudinal Data. 2nd ed. Oxford University Press; 2002.

17. Schnack HG, van Haren NE, Hulshoff Pol HE, et al. Reliability of brain volumes from multicenter MRI acquisition: a calibration study. Hum Brain Mapp. 2004;22(4):312-320.

18. Devi CN, Chandrasekharan A, Sundararaman VK, Alex ZC. Neonatal brain MRI segmentation: A review. Comput Biol Med. 2015;64:163-178.

19. Ding Y, Acosta R, Enguix V, et al. Using deep convolutional neural networks for neonatal brain image segmentation. Front Neurosci. 2020;14:207.

20. Makropoulos A, Counsell SJ, Rueckert D. A review on automatic fetal and neonatal brain MRI segmentation. Neuroimage. 2018;170:231-248.

21. Gaser C, Dahnke R. CAT - A computational anatomy toolbox for the analysis of structural MRI data. Accessed April 13, 2021. http://www.neuro.uni-jena.de/hbm2016/ GaserHBM2016.pdf

22. Gu C. Smoothing Spline ANOVA Models. Springer; 2002.

23. Rigby RA, Stasinopoulos DM. Generalized additive models for location, scale and shape. J R Stat Soc Ser C Appl Stat. 2005;54(3):507-554.

24. Rigby RA, Stasinopoulos DM. Smooth centile curves for skew and kurtotic data modelled using the Box-Cox power exponential distribution. Stat Med. 2004;23(19):3053-3076.

25. Hüppi PS, Warfield S, Kikinis R, et al. Quantitative magnetic resonance imaging of brain development in premature and mature newborns. Ann Neurol. 1998;43(2):224-235.

26. Makropoulos A, Robinson EC, Schuh A, et al. The Developing Human Connectome Project: a minimal processing pipeline for neonatal cortical surface reconstruction. Neuroimage. 2018;173:88-112.

27. Borghi E, de Onis M, Garza C, et al. Construction of the World Health Organization child growth standards: selection of methods for attained growth curves. Stat Med. 2006;25(2):247-265. 
28. Narvacan K, Treit S, Camicioli R, et al. Evolution of deep gray matter volume across the human lifespan. Hum Brain Mapp. 2017;38(8):3771-3790.

29. Thompson DW. On Growth and Form. Cambridge University Press; 1917.

30. Kilgour AHM, Todd OM, Starr JM. A systematic review of the evidence that brain structure is related to muscle structure and their relationship to brain and muscle function in humans over the lifecourse. BMC Geriatr. 2014;14:85.

31. Mandell JG, Hill KL, Nguyen DTD, et al. Volumetric brain analysis in neurosurgery: Part 3. Volumetric CT analysis as a predictor of seizure outcome following temporal lobectomy. $J$ Neurosurg Pediatr. 2015;15(2):133-143.

32. Lynch KM, Shi Y, Toga AW, Clark KA. Hippocampal shape maturation in childhood and adolescence. Cereb Cortex. 2019;29(9):3651-3665.

33. Reiss AL, Abrams MT, Singer HS, et al. Brain development, gender and IQ in children. A volumetric imaging study. Brain. 1996;119(Pt 5):1763-1774.

34. Roche AF, Mukherjee D, Guo SM, Moore WM. Head circumference reference data: birth to 18 years. Pediatrics. 1987;79(5):706-712.

35 Kuczmarski RJ, Ogden CL, Grummer-Strawn LM, et al. CDC growth charts: United States. Adv Data. 2000;(314):1-27.

36. de Onis M, C Garza, AW Onyango, Martorell R. WHO child growth standards. Acta Paediatr. 2006;95(Suppl 450):1-101.

37. Morton SG. Crania Americana: A Comparative View of the Skulls of Various Aboriginal Nations of North and South America. J Dobson; 1839.

38. Nopoulos P, Flaum M, O'Leary D, Andreasen NC. Sexual dimorphism in the human brain: evaluation of tissue volume, tissue composition and surface anatomy using magnetic resonance imaging. Psychiatry Res. 2000;98(1):1-13.

39. Lenroot RK, Gogtay N, Greenstein DK, et al. Sexual dimorphism of brain developmental trajectories during childhood and adolescence. Neuroimage. 2007;36(4):1065-1073.

40. Holland D, Chang L, Ernst TM, et al. Structural growth trajectories and rates of change in the first 3 months of infant brain development. JAMA Neurol. 2014;71(10):1266-1274.

41. Sowell ER, Peterson BS, Thompson PM, et al. Mapping cortical change across the human life span. Nat Neurosci. 2003;6(3):309-315.

42. Benes FM, Turtle M, Khan Y, Farol P. Myelination of a key relay zone in the hippocampal formation occurs in the human brain during childhood, adolescence, and adulthood. Arch Gen Psychiatry. 1994;51(6):477-484.

43. Sowell ER, Thompson PM, Toga AW. Mapping changes in the human cortex throughout the span of life. Neuroscientist. 2004;10(4):372-392.
44. Cowan WM, Fawcett JW, O'Leary DD, Stanfield BB. Regressive events in neurogenesis. Science. 1984;225(4668):12581265.

45. Decker AL, Duncan K, Finn AS, Mabbott DJ. Children's family income is associated with cognitive function and volume of anterior not posterior hippocampus. Nat Commun. 2020;11(1):4040.

\section{Disclosures}

The authors report no conflict of interest concerning the materials or methods used in this study or the findings specified in this paper.

\section{Author Contributions}

Conception and design: Schiff, Peterson. Acquisition of data: Peterson, Cherukuri. Analysis and interpretation of data: all authors. Drafting the article: Schiff, Peterson, Paulson. Critically revising the article: all authors. Reviewed submitted version of manuscript: all authors. Approved the final version of the manuscript on behalf of all authors: Schiff. Statistical analysis: Peterson. Study supervision: Schiff.

\section{Supplemental Information Online-Only Content}

Supplemental material is available with the online version of the article.

Supplemental Material. https://thejns.org/doi/suppl/10.3171/ 2021.2.PEDS201006.

\section{Previous Presentations}

This work was previously presented at the 48th Annual Meeting of the AANS/CNS Section on Pediatric Neurological Surgery held in Phoenix, Arizona, on December 5-8, 2019.

\section{Preprint Server}

An earlier version of this article can be found on a preprint server. Preprint server name: medRxiv.

Preprint DOI: 10.1101/2020.05.19.20102319.

\section{Correspondence}

Steven J. Schiff: Penn State University, University Park, PA. steven.j.schiff@gmail.com. 